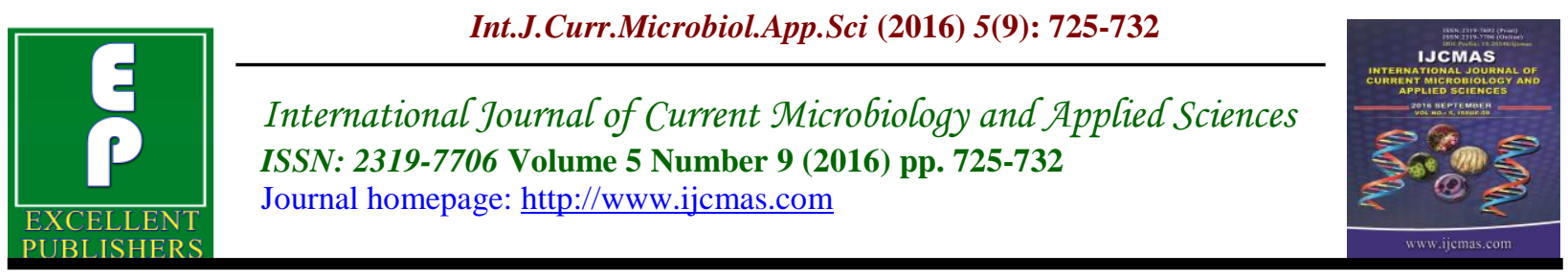

Original Research Article

http://dx.doi.org/10.20546/ijcmas.2016.509.083

\title{
Clinical Profile and Diagnostic Parameters of Dengue Viral Infection among Children
}

\author{
Asmabegaum M. Biradar ${ }^{*}$, Shobha D. Nadagir ${ }^{2}$, \\ Mahesh Kumar Shankar ${ }^{3}$ and Trupti B. Naik ${ }^{4}$ \\ ${ }^{1}$ Al- Ameen Medical College, Athani road, Vijayapur, 586109 \\ Karnataka, India \\ ${ }^{2}$ Navodaya Medical College, Raichur, Karnataka, India \\ ${ }^{3}$ Karnataka institute of Medical Sciences, Hubballi, Karnataka, India \\ ${ }^{4}$ Subbaiah Institute of Medical Sciences, Shivamogga., Karnataka, India \\ *Corresponding author
}

\begin{abstract}
A B S T R A C T
Keywords

Dengue, IgM antibody, NS1, platelet count, IC test.

\section{Article Info}

Accepted:

28 August 2016

Available Online:

10 September 2016

Dengue fever is an acute febrile arbovirus disease. Dengue is almost endemic all over India. Complications caused are dengue hemorrhagic fever (DHF) and dengue shock syndrome(DSS).Present study was conducted to know the sero prevalence of dengue infection and correlate clinical manifestations and diagnostic parameters of dengue infection in children. We conducted a cross sectional study on patients with acute febrile illness, clinically diagnosed as having dengue fever as per the WHO criteria. Serum samples collected from these patients were tested for Detection of IgM antibody by MAC ELISA and immune chromatographic (IC) test. Detection of NS1 antigen by IC test. Detection of platelet count by automated machine. Among 200 dengue suspected cases 37(18.5\%) were positive by IgM MAC ELISA, 22(11\%) by IgM IC test and $12(6 \%)$ by NS1 antigen IC test, of which 7(3.5\%) were positive by both IgM antibody and NS1antigen. Among seropositive, 35 (83.33\%) revealed thrombocytopenia. Maximum children 27 (64.28\%) presented with bleeding manifestation. However, no significant difference observed with hematocrit values. Prevalence of dengue infection was $21 \%$. Most of them (59.53\%) presented with DHF. Significant association of thrombocytopenia found in dengue positive subjects, more so when NSI antigen and Ig M antibody are detected simultaneously.
\end{abstract}

\section{Introduction}

Dengue virus, a flavivirus responsible for dengue fever. Infection with dengue virus causes wide array of clinical illness from mild viral syndrome, classical dengue fever which can turn into fatal hemorrhagic disease. Dengue hemorrhagic fever (DHF) as well as dengue shock syndrome(DSS) are the complications caused by dengue virus (Gubler et al., 1998).

Dengue is almost endemic all over India. Confirmation of Dengue viral infection is 
most important and essential pre-requisite for the managing complications associated with Dengue viral infection (Kulkarni et al., 2010).

Current diagnostic methods used are 1) viral culture 2) viral RNA detection by reverse transcriptase polymerase chain reaction(RTPCR) and 3) serological tests. Detection of IgM immunoglobulin and NS 1 antigen are routine serological tests used for diagnosis of acute Dengue viral infection (Datta et al., 2010).

Dengue NS1 antigen identification is possible as early as day one of onset of fever, as it appears in serum in initial phase of infection. Dengue $\operatorname{IgM}$ immunoglobulin usually starts appearing in serum 2-5 days after the infection. Hence pooling both results of Dengue IgM antibody and NS1 antigen gives a more accurate diagnosis in acute phase. Apart from specific markers of Dengue virus, platelet count is the sole supportive test which is in reach of many laboratories mainly in peripheral areas (Badave et al., 2015).

Present study has been done to know the sero prevalence of dengue viral infection among children in a tertiary care health center. An attempt was made for correlating clinical manifestations along withdiagnostic parameters of dengue viral infection.

\section{Materials and Methods}

Cross sectional evaluation was conducted during the period, December 2010 to November 2011 at department of Microbiology, Karnataka Institute of Medical Sciences, Hubballi. Inclusion criteria: Patients admitted in Karnataka Institute of Medical Sciences Hospital Hubballi, with acute febrile disorder, clinically diagnosed as having dengue viralfever as per to the WHO criteria.
Exclusion criteria: Patients with history of prolonged fever of more than one month and patients with any other proven febrile illnesses like Malaria, Typhoid etc.

Detailed history obtained from each patient regarding duration of illness and other symptoms associated with fever. Clinical aspects and relevant examination finding were recorded. Standard aseptic precautions were used to collect 5 to $8 \mathrm{~mL}$ of blood from each patient. And same was distributed in two aliquots.

One portion- 2 to $3 \mathrm{~mL}$ of blood collected in a tube having EDTA was used forHb\%., total count, differential count, peripheral smear, platelet count and hematocrit values.

Second portion- 3 to $5 \mathrm{~mL}$ collected in a plain tube (Without anticoagulant). Serum was separated by centrifugation. Serum samples were subjected to detection of $\operatorname{IgM}$ antibody by using MAC ELISA obtained from- "Dengue-IgM antibody capture ELISA" (Arbovirus Diagnostics, NIV, Pune, India) and ImmunoChromatographic Test (ICT). NS1 antigen was identified by using ICT.

Kit Used: "Dengue DAY 1 Test -Rapid visual test for the detection of Dengue NS1 antigen and differential detection of $\operatorname{IgM}$ and IgG antibodies in Human Serum/ Plasma. J. Mitra \& Co. Private Limited

Controls: A total of 40 serum samples were collected as control. Of these twenty serum samples were from patients with fever due to a proven aetiology other than dengue infection. Other twenty serum samples were from healthy individuals.

\section{Results and Discussion}

Total of 200 serum samples were gathered from patients who were suffering from fever 
suspected to have dengue infection. Dengue positivity was seen in 42 (21\%) patients. IgM antibody positivity was seen in 37 $(18.5 \%)$ patients, $12(6 \%)$ patients were positive for NS1 antigen.

More number of dengue viral positivity was seen in the, month of June and September.Majority of patients $23(54.76 \%)$ belonged to rural area. Age group was ranging from five months to 14 years, $61.90 \%$ were males and $38.09 \%$ were females.

NS1 was positive in $5(11.90 \%)$ cases. $30(71.42 \%)$ cases were positive for IgM by MAC ELISA only, 22(11\%) cases were positive for $\operatorname{IgM}$ by IC card test only, $7(16.66 \%)$ cases were positive for both NS1 antigen and IgM MAC ELISA. Maximum number that is $30(71.42 \%)$ were diagnosed through IgM MAC Elisa in our study. All the $22(11 \%)$ samples positive by IgM IC card test were also positive by IgM MAC ELISA. That shows ELISA is more sensitive than IC card test in diagnosing IgM antibody.

All patients were suffering from febrile illness $100 \%$. Majority of patients had associated myalgia $64.28 \%$. $64.28 \%$ of dengue positive cases presented with bleeding manifestations. Whereas only $19.62 \%$ of dengue negative patients were having the bleeding manifestations. The difference is statistically significant. $57.14 \%$ of dengue positive cases were having hepatosplenomegaly. There was no statistically significant difference between dengue positive and dengue negative cases.

Maximum patients $31(25.25 \%)$ who were positive for $\operatorname{IgM}$ antibody had duration of fever ranging from 6-12 days. Only 3(4.68\%) cases were positive for IgM antibody in first five days. All the 7 cases positive for NS1 in first five days of fever.
Of total 200 patients $43.5 \%$ had thrombocytopenia. The platelet range of 20,000-50,000 was observed among 26(13\%) of total patients. $11(5.5 \%)$ of dengue seropositive cases have that range. Among dengue seropositive patients majority $(88.33 \%)$ revealed thrombocytopenia. The difference is statistically significant. All seven patients $(100 \%)$ with NSI antigen and $\operatorname{IgM}$ antibody both positive, one (20\%) patent with NS1 antigen positive only and $27(90 \%)$ with Ig $\mathrm{M}$ antibody positive only revealed platelet count less than 100000.

High hematocrit values were observed in only two $(8 \%)$ children suffering from DHF.

In comparison with IgM MAC ELISA, Sensitivity and Specificity of IgM Card test was $67.56 \%$ and $100 \%$ respectively. Positive predictive value $100 \%$ and negative predictive value $93.14 \%$

\section{Control group}

Serum samples of all twenty healthy individuals and twenty febrile patients (Malaria -9patients, Typhoid -11patients) tested for NS1 antigen, IgM IC card and IgM MAC ELISA were found to be negative

We conducted a cross sectional study on caseswith acute febrile illness, clinically diagnosed as having dengue viral fever as per as the WHO criteria.

A total of 200 serum samples from cases were subjected to three different tests in the diagnosis of dengue viral infection such as Detection of $\operatorname{IgM}$ antibody by $\operatorname{IgM}$ MAC ELISA and ICT, Detection of NS1 antigen by ICT.

Inclusion of ICT and ELISA both in our study has given us an opportunity to compare 
efficacy of ICT in diagnosis of Dengue infection. Detection by ICT offers a rapid method for presumptive diagnosis of dengue infection. Based on these test results prevalence of dengue viral infection in this study was $42(21 \%)$. The present study is comparable with Piyush et al., 2008; Jigna et al., 2011; Atul et al., 2011).

Ratio of male to female was $1.625: 1$ similar with study by (Dash et al., 2005) and (Neerja et al., 2006). Age group of majority of dengue positive cases was 5-10 years constituting $73.8 \%$. Youngest patient was 5 months old. Our findings are in agreement with the report by (Dar et al., 1999) and (Atul et al., 2011). Some of the studies showed most common age group as 5-15 years (Shahet al., 2006).

Maximum number $(54.76 \%)$ of cases belonged to rural area. Piyush et al., (2008) also quoted higher $(53.41 \%)$ prevalence of dengue viral infection among rural population.

We noticed seasonal variation. Data was analyzed on monthly basis. Incidence was high in the rainy season. The correlation between incidence of dengue viral infection with monsoon season was clearly evident in the present study and is supported by similar observations from different regions such as (Lal et al., 2007) (Kumar et al., 2010).

Out of 200 total study population $37(18.5 \%)$ were positive by IgM MAC ELISA and $22(11 \%)$ were positive for IgM antibody by ICT. Of 42 dengue positive cases 37(88.09\%) were detected by IgM capture ELISA and 22 $(52.38 \%)$ were detected by IgM ICT card test. Datta et al., (2010) quoted overall positivity as $39.1 \%$. They have shown that NS1 was positive in 140 out of $600(23.3 \%)$. The entire $22(100 \%)$ samples positive for IgM IC card test were also positive by IgM MAC ELISA as shown in Table.2. IgM MAC ELISA has more sensitivity as compared with IgM IC card test. Moorthy et al., (2009) also stated higher sensitivity with IgM MAC ELISA as compared to rapid tests. Kulkarni et al., (2010)quoted over all positivity rate as $15 \%$. IgM antibody was positive in $50 \%$ of cases in their study they used ICT as diagnostic tool. Sensitivity of IgM ICT in comparison with IgM MAC ELISA was $67.56 \%$ and Specificity $100 \%$. Satish et al., (2002) quoted sensitivity of ICT as $73 \%$ and IgM MAC ELISA as $96 \%$.

The clinical study of dengue viral infection revealed that fever was the most frequent presenting symptom. As ours is a tertiary care hospital, patients coming to our center are generally referred from different peripheral centers. Hence most of the patients reach here at the end of first week of illness. Among these patients $8(19.04 \%)$ had fever ranging from 1-5 days, $31(73.80 \%)$ had fever ranging from 6-12days and $3(7.14 \%)$ had fever duration more than 12 days. As shown in Table. 3 Many other studies have substantiated fever as most common presenting symptom (Dash et al., 2005), (Khan et al., 2007).

Majority $64.28 \%$ cases had associated bleeding manifestations in the form of petechiae, bleeding gums, skin rash. The most common bleeding manifestation in the present study was petechiae $(61.90 \%)$. In comparison with Dengue negative cases (19.62\%) bleeding manifestations were noted in significantly more number of Dengue seropositive cases (64.28\%).

Majority of patients had associated myalgia $64.28 \%$. Other symptoms were, vomiting, skin rash, headache, facial puffiness, altered sensorium and hepatosplenomegaly which is in concordance with study done by (Ashwin et al., 2010). Hepatosplenomegaly was found among57.14\% of dengue positive cases. 
Statistically significant difference was not there between dengue positive and dengue negative cases. As shown in Table. 2

Thrombocytopenia was seen is $88.33 \%$ of positive cases. Maximum that is $46.5 \%$ patient had platelet count in the range of
51000- 100000. In correlation with dengue sero negative patient's association of thrombocytopenia was statistically significant. This feature is in agreement with many other studies (Cherian et al., 1994; Neeraja et al., 2006).

Table.1 Dengue specific parameters in the diagnosis of dengue infection

\begin{tabular}{|l|c|l|}
\hline Parameters & Total number & Percentage \% \\
\hline NS1 antigen only & 5 & 11.90 \\
\hline IgM by MAC ELISA only & 30 & $71.42 \%$ \\
IgM IC card test only & 22 & $11 \%$ \\
\hline $\begin{array}{l}\text { Both NS1 antigen and IgM by } \\
\text { MAC ELISA }\end{array}$ & 7 & $16.66 \%$ \\
\hline Total & 42 & 100 \\
\hline
\end{tabular}

Table.2 Comparison of clinical manifestation between dengue seropositive and seronegative cases.

\begin{tabular}{|l|l|l|l|}
\hline Clinical features & $\begin{array}{l}\text { Dengue } \\
\text { negative Cases } \\
(\mathrm{n}=158)\end{array}$ & $\begin{array}{l}\text { Dengue } \\
\text { positive cases } \\
(\mathrm{n}=42)\end{array}$ & $\mathrm{P}$ value \\
\hline Head ache & $34(21.51 \%)$ & $13(30.95 \%)$ & 0.0011 \\
\hline Myalgia & $96(60.75 \%)$ & $27(64.28 \%)$ & 0.0 \\
\hline Vomiting & $75(47.46 \%)$ & $22(52.38 \%)$ & 0.0 \\
\hline Skin rash & $47(29.74 \%)$ & $15(35.71 \%)$ & 0.00001 \\
\hline Altered sensorium & $11(6.98 \%)$ & $2(4.76 \%)$ & 0.011 \\
\hline Facial puffiness & $19(12.02 \%)$ & $7(16.66 \%)$ & 0.014 \\
\hline $\begin{array}{l}\text { Bleeding } \\
\text { manifestations }\end{array}$ & $31(19.62 \%)$ & $27(64.28 \%)$ & 0.0001 \\
\hline Icterus & $4(2.53 \%)$ & $1(2.38 \%)$ & 0.9557 \\
\hline Hepatosplenomegaly & $75(47.46 \%)$ & $24(57.14 \%)$ & 0.2650 \\
\hline Hepatomegaly & $29(18.35 \%)$ & $10(23.8 \%)$ & 0.4277 \\
\hline Splenomegaly & $5(3.16 \%)$ & $2(4.76 \%)$ & 0.6166 \\
\hline
\end{tabular}

Table.3 Comparison of duration of fever with different dengue parameters

\begin{tabular}{|l|l|l|l|}
\hline $\begin{array}{l}\text { Duration of illness } \\
\text { in days }\end{array}$ & $\begin{array}{l}\text { NS1 antigen } \\
\text { positive cases }\end{array}$ & $\begin{array}{l}\text { IgM ELISA positive } \\
\text { cases }\end{array}$ & $\begin{array}{l}\text { IgM IC card positive } \\
\text { cases }\end{array}$ \\
\hline 1-5days $(\mathrm{n}=64)$ & $7(10.93 \%)$ & $3(4.68 \%)$ & $1(1.56 \%)$ \\
\hline 6-12days $(\mathrm{n}=123)$ & $5(4.06 \%)$ & $31(25.205)$ & $21(17.07 \%)$ \\
\hline$>12$ days $(\mathrm{n}=13)$ & 0 & $3(23.07 \%)$ & 0 \\
\hline Total $\mathrm{n}=200$ & $12(6 \%)$ & $37(18.5 \%)$ & $2211 \%)$ \\
\hline
\end{tabular}


Table.4 Comparison of Platelet count between dengue seropositive and seronegative cases

\begin{tabular}{|l|l|l|l|}
\hline & $\begin{array}{l}\text { Dengue negative } \\
\text { cases n=158 }\end{array}$ & $\begin{array}{l}\text { Dengue } \\
\text { positive cases } \\
\mathrm{n}=42\end{array}$ & P value \\
\hline$<20,000$ & $2(1.26 \%)$ & $1(2.38 \%)$ & 0.5972 \\
\hline $20,000-50,000$ & $15(9.49 \%)$ & $11(26.19 \%)$ & 0.0042 \\
\hline $51,000-1,00,000$ & $70(44.30 \%)$ & $23(54.76 \%)$ & 0.2271 \\
\hline$>1.00,000$ & $71(44.93 \%)$ & $7(16.66 \%)$ & 0.0080 \\
\hline
\end{tabular}

Table.5 Comparison of dengue IgM MAC ELISA results with IgM IC card test

\begin{tabular}{|l|l|l|l|}
\hline \multirow{2}{*}{ IgM IC card test } & \multicolumn{2}{|l|}{ Dengue IgMMAC ELISA } & Total \\
\cline { 2 - 3 } & Dengue-positive & Dengue-negative & \\
\hline Dengue-positive & 22 & 0 & 22 \\
\hline Dengue-negative & 15 & 163 & 178 \\
\hline Total & 37 & 163 & 200 \\
\hline
\end{tabular}

Only $8 \%$ of cases with DHF had high hematocrit concentration. In contrast to study done by Shah et al., (2006) who stated $100 \%$ of DHF/DSS as having high hematocrit concentration.

We could detect Dengue Specific IgM antibody from $5^{\text {th }}$ day of fever onwards. $31(73.80 \%)$ dengue positive patients detected by IgM MAC ELISA had history of fever ranging from 6-12 days, only $7.14 \%$ of patients had duration of fever ranging from 15day and more than 12 days. As shown in Table.3. This is in agreement with Sophie et al., (2002).

Out of 42 dengue seropositive cases 12 (28.57 $\%)$ were detected by NS1 antigen IC card test. A large number of cases would be missed if NS1 is not included inthe test panel (Han et al., 2004).
We tried to find the association of dengue parameter positivity with thrombocytopenia. Of the 42 cases, 35 (83.33\%) showed thrombocytopenia. As shown in table.4. Thrombocytopenia was noted in 27 (90\%) patients with only $\operatorname{IgM}$ antibody positive result. Further analysis of two groups NS1 only $(20 \%)$ v/s NS1 plus IgM (100\%) revealed that thrombocytopenia was associated predominantly when both NS1 and IgM were positive.

Most patients (59.53\%) were suffering from dengue hemorrhagic fever (DHF). No case of dengue shock syndrome was noticed in this study. Other studies have quoted dengue hemorrhagic fever ranging from 5\%-60\%, dengue shock syndrome ranging from $1 \%$ $17.3 \%$ (Neeraja et al., 2006). 
Majority $(85.71 \%)$ of dengue positive patients responded to symptomatic treatment. Only $6(14.28 \%)$ of them received blood products as a part of treatment. One patient (2.38\%) received whole blood transfusion, three (7.14\%) patients fresh frozen plasma and two $(4.76 \%)$ patients platelet transfusion. Two cases who received platelet transfusion had platelet count below 20000. Mortality was not noted in any dengue seropositive cases.

In conclusion, overall prevalence of dengue infection was $21 \%$. Most of them (59.53\%) presented with DHF. We foundIgM MAC ELISA to be more sensitive in comparison with IC test. It is essential to use both parameters NSI antigen and IgM antibody detection for early diagnosis and proper management of dengue infection.

\section{References}

Ashwin, K., Chythra, R. R., Vinay, P., Seema, S., Chanaveerappa, B. and Charmaine M. S. 2010. Clinical Manifestation and trends of Dengue cases admitted in a tertiary care hospital, Udupi District, Karnataka. Indian J. Community Med., 35(3): 86-390.

Atul, G., Jaya, G., Rao, Y.K., Upadhyay, G. C. and Suman, S. 2011. Prevalence of

Badave, K.G., Sai, S.P., Rao P.N. 2015. Importance of NS1 antigen detection and its association with platelet count for early diagnosis of dengue virus infection. Int. J. Curr. Microbiol. Appl. Sci., 4: 779-784.

Cherian, T., Ponnuraj, E., Kuruvilla, T., Chellam, K., John, T.J. and Raghupathy, P. 1994. An epidemic of dengue hemorrhagic fever and dengue shock syndrome in and around Vellore. Indian J. Med. Res., 100: 5156.

Dar, L., Broor, S., Sengupta, S., Xess, I. and Seth, P. 1999. The first major outbreak of dengue haemorrhagic fever in Delhi, India. Emerg. Infect. Dis., 5: 58990.

Dash, P.K., Saxena, P., Abhavankar, A., Bhargava, R. and Jana, A.M. 2005. Emergence of dengue virus type 3 in Northern India. Southeast Asian J. Trop. Med. Public Health, 36: 370-77.

Datta, S., Wattal, C. 2010. Dengue NS1 antigen detection: A useful tool in early diagnosis of dengue virus infection. Indian J. Med. Microbiol., 28: 107-10.

dengue among clinically suspected febrile episodes at a teaching hospital in North India. J. Infect. Dis. Immunol., 3: 85-89.

Gubler, D.J., Roehrig, J.T. Arboviruses (Togaviridae and Flaviviriadae) In: Mahy, B.W.J., Collier, L. 1998. Topley and Wilson's Microbiology and Microbial infections, London 9: 579-600.

Hang, T.V., Nguyet, M.N., Trung, Tricou, V., Yoksan, S., Minh, N.D. 2009. Diagnostic Accuracy of NS1 ELISA and Lateral Flow Rapid Tests for Dengue sensitivity, Specificity and Relationship to Viraemia and Antibody Responses. PLOS Neglected Trop. Dis., 3: 360.

Jigna, K., Parul, P., Jignasa, B., Hitendra, B., Chirag Patel. 2011. Evaluation of commercial newer rapid test for detection of early acute dengue infection. Nat. J. Med. Res., 2: 2249.

Khan, E., Siddiqui, J., Shakoor, S., Mehraj, V., Jamil, B., Hasan, R. 2007. Dengue outbreak in Karachi, Pakistan, Transaction of Royal Soc. Trop. Med. Hygiene, 101: 1114-9.

Kulkarni, R.D., Patil, S.S., Ajantha, G.S., Upadhya, A.K., Kalabhavi, A.S., Shubhada, R.M., Shetty, P.C. 2010. Association of platelet count and serological markers of dengue 
infection-importance of NS1 antigen. Indian J. Med. Microbiol., 29: 359-362.

Kumar, A., Rao, R., Pandit, V., Shetty, S., Bamigatti, C., Samaraging, C.M. 2010. Clinical manifestation and trend of dengue cases admitted in tertiary care hospital, udupi, Karnataka. Indian J. Community Med., 35: 386-391.

Lal, M., Aggarwal, A., Oberoi, A. 2007. Dengue Fever- An emerging viral fever in Ludhiana, North India. Indian J. Public Health, 51: 198-9.

Moorthy, M., Chandy, S., Selvaraj, K., Abraha, A.M. 2009. Evaluation of a rapid immunocromatographic device for the detection of $\operatorname{IgM} \& \operatorname{IgG}$ antibodies to dengue viruses (DENV) in a tertiary care hospital in South India. Indian J. Med. Microbiol., 27: 254-6.

Neeraja, M., Lakshmi, V., Teja, V. D., Umabala, P. and Subbalakshmi, M. V. 2006. Serodiagnosis of dengue virus infection in patients presenting to a tertiary care hospital. Indian J. Med. Microbiol., 24: 280-2.

Piyush, T., Rashmi, K., Sanjeev T., Tambe, J.J. and Vimala, V. 2008. Descriptive Epidemiology of Dengue Transmission in Uttar
Pradesh. Indian Pediatrics, 45: 315318.

Sathish, N., Manayani D.J., Shankar V., Abraham, M., Nithyanandam G., Sridharan, G. 2002. Comparison of IgM capture ELISA with a commercial rapid immunochromatographic card test \& $\operatorname{IgM}$ microwell ELISA for the detection of antibodies to dengue viruses Indian J. Med. Res., 115: 31-6.

Shah, G.S., Islam, S., Das, B.K. 2006. Clinical and laboratory profile of dengue infection in Children. Kathmandu Univ. Med. J., 13: 40-4.

Sing, N.P., Jhamb, R., Agarwal, S.K., Gaiha, M., Dewan, R., Daga, M.K. 2005. The 2003 outbreak of dengue fever in Delhi, India. South east Asian J. Trop. Med. Public Health, 36(12): 1174-78.

Sophie, A.A., Monique, D., Andrew, F., Vincent, D., Marie, F. 2002. Enzymelinked immunosorbent assay specific to dengue virus type 1 nonstructural protein ns1 reveals circulation of the antigen in the blood during the acute phase of disease in patients experiencing primary or secondary infections. J. Clin. Microbiol., 409(2): 376-381.

\section{How to cite this article:}

Asmabegaum M. Biradar, Shobha D. Nadagir, Mahesh Kumar Shankar and Trupti B. NaiK. 2016. Clinical Profile and Diagnostic Parameters of Dengue Viral Infection among Children. Int.J.Curr.Microbiol.App.Sci. 5(9): 725-732. doi: http://dx.doi.org/10.20546/ijcmas.2016.509.083 